\title{
W Polarisation and \\ Spin Density Matrix Measurements at LEP
}

\author{
Helge Voss, \\ IPHE Lausanne
}

OPAL Collaboration, on behalf of the LEP experiments

- Introduction

- Polarised Cross Section

- SDM measurements

- Spin Correlations

- CP Test

- Summary 


\section{Why Studying W Polarisation ?}

- model independent approach to Triple Gauge Couplings:

$\leftrightarrow$ anomalous TGCs change W-helicity distributions

see EW-2 S. Jezequel

- longitudinal component of W-Bosons

$\leftrightarrow$ electroweak symmetry breaking

e.g. new physics with composite W's

$\longrightarrow$ other mass generation mechanism

$\longrightarrow$ anomalous electric, magnetic moment (aTGCs) 


\section{What's it all about}

- W-Bosons: 3 spin (helicity) states

- observe individually and in correlation

$$
\begin{aligned}
& \Longrightarrow \tau=+1 \\
& \dddot{\tau} \tau=0 \\
& \Longleftrightarrow \tau=-1
\end{aligned}
$$

- helicity content depends on W-production angle $\cos \theta_{\mathrm{W}}$

LEP: $\mathrm{e}^{+} \mathrm{e}^{-} \rightarrow \mathrm{W}^{+} \mathrm{W}^{-}$
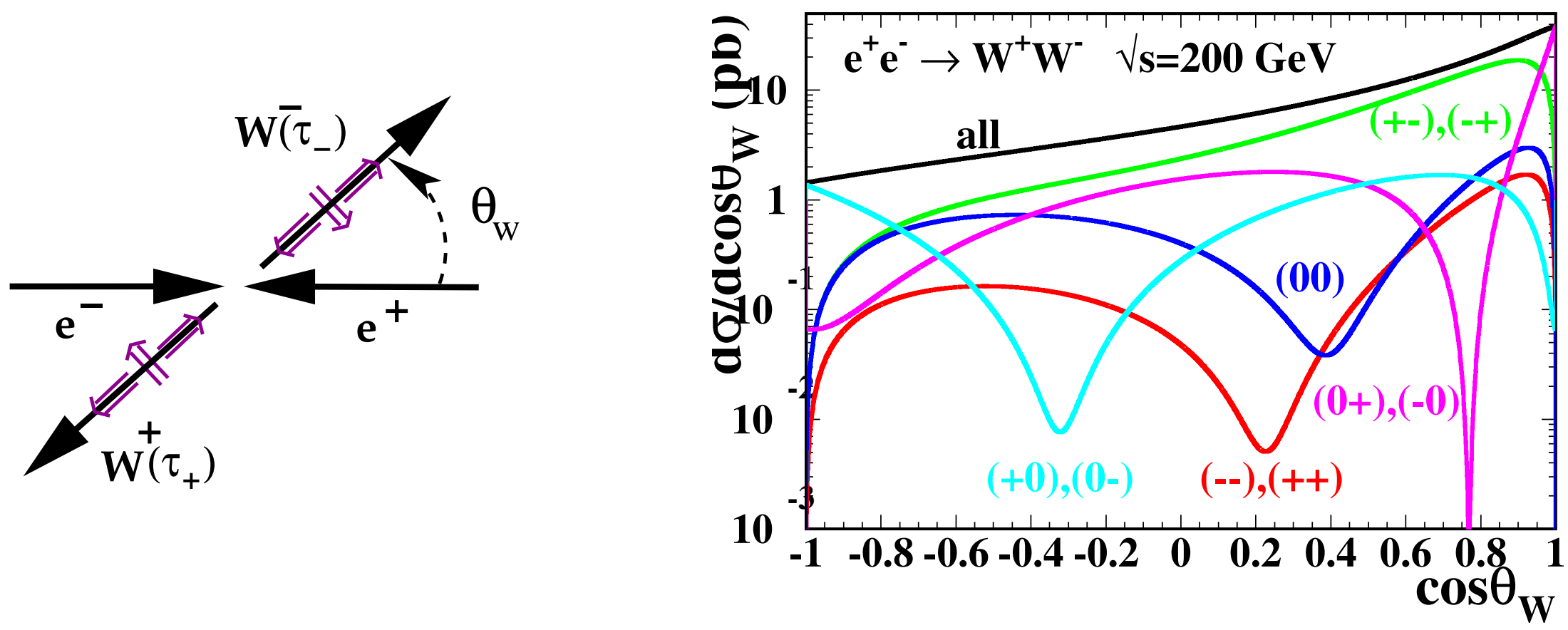


\section{W Polarisation - Signatures}

- V-A structure of W decay reflects polarisation

- $\cos \theta^{*}$ : polar angle in $\mathrm{W}$ rest frame of charged lepton, d-type quark

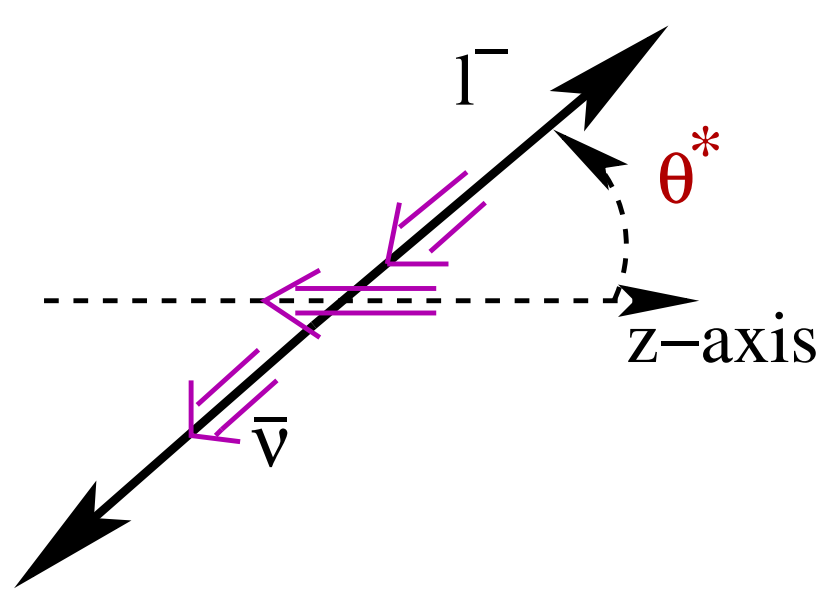

decay distribution in $\mathrm{W}$ rest-frame

\begin{tabular}{c|c}
$\mathrm{W}^{-}$-helicity & $\begin{array}{c}\text { decay } \\
\text { distribution }\end{array}$ \\
\hline-1 & $\frac{3}{8}\left(1+\cos \theta^{*}\right)^{2}$ \\
+1 & $\frac{3}{8}\left(1-\cos \theta^{*}\right)^{2}$ \\
0 & $\frac{3}{4} \sin ^{2} \theta^{*}$
\end{tabular}

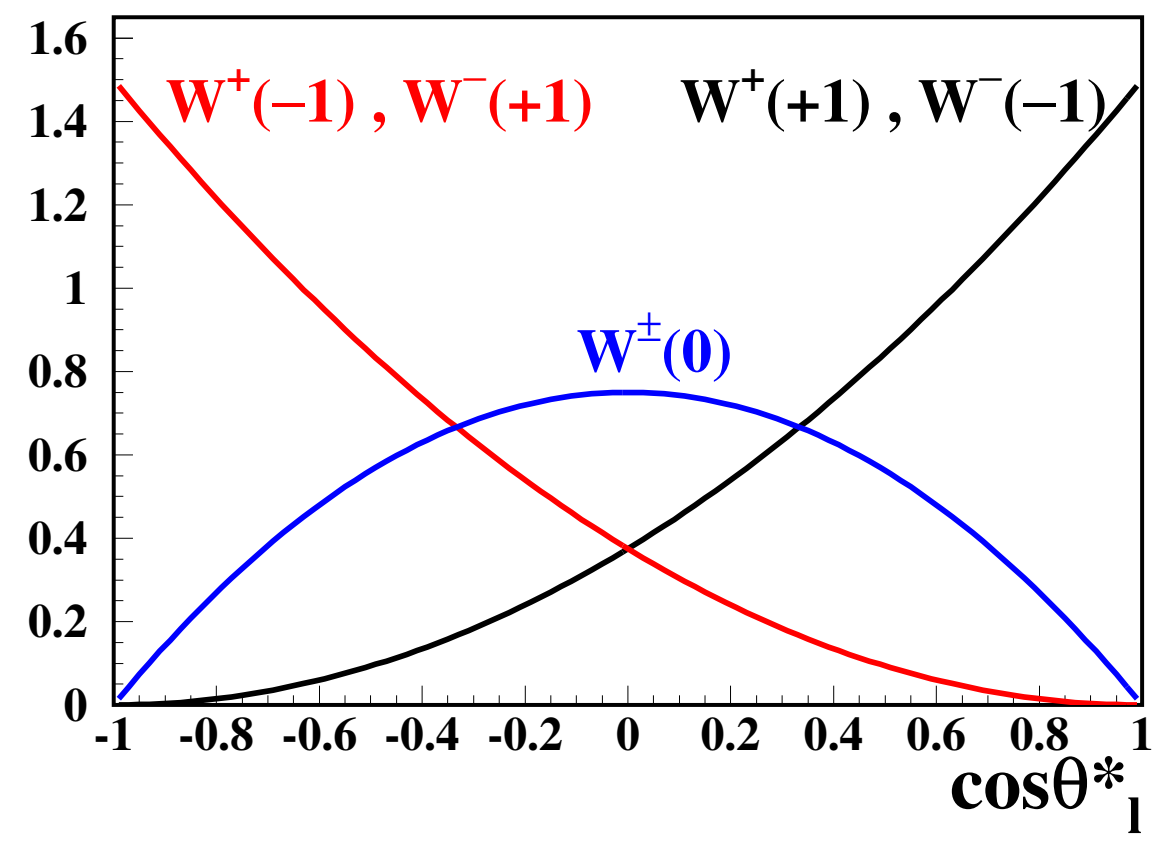




\section{W Polarisation - Experimental Access}

- $\mathrm{WW} \rightarrow \mathrm{q} \overline{\mathrm{q}} \mathrm{q} \overline{\mathrm{q}}, \mathrm{q} \overline{\mathrm{q}} \ell \bar{\nu}_{\ell}, \ell \bar{\nu}_{\ell} \bar{\ell}^{\prime} \nu_{\ell^{\prime}}$

use: $\mathrm{W}^{+} \mathrm{W}^{-} \rightarrow \mathrm{q} \overline{\mathrm{q}} \ell \bar{\nu}_{\ell}$

- identification of $\mathbf{W}$

- $\cos \theta_{\ell}^{*}$ without ambiguity
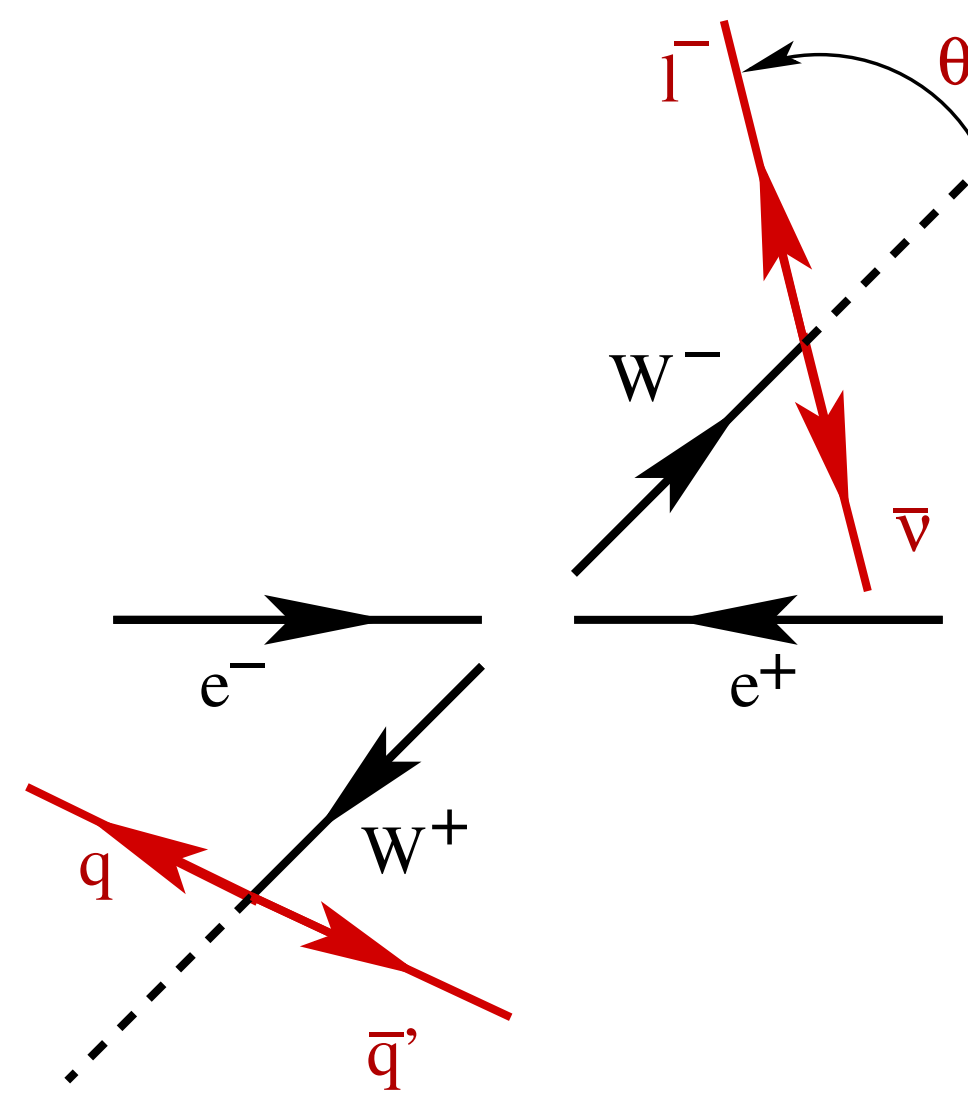

access to helicities:

- $\mathrm{W} \rightarrow \ell \nu: \Rightarrow \tau=(-1),(+1),(0)$

- $\mathrm{W} \rightarrow \mathrm{q} \overline{\mathrm{q}}: \Rightarrow \tau=( \pm 1)$, 


\section{Polarisation - SDM measurements}

- direct measurement (L3) $\sqrt{s}=(183-208) \mathrm{GeV}$

$\rightarrow$ fit analytical helicity distribution to $\cos \theta^{*}$

- Spin Density Matrix (SDM) (DELPHI, L3, OPAL) $\sqrt{s}=189 \mathrm{GeV}$ $\longrightarrow$ projection onto "squares" of helicity amplitudes

Comparison with theoretical distributions:

bin by bin correction for efficiency and bin-migration 


\section{Longitudinal W Helicity Fractions}

longitudinally polarised W-Bosons

$\mathrm{L} 3 \sqrt{s}=(183-208) \mathrm{GeV}$

DATA $0.228 \pm 0.027 \pm 0.012$

expected

0.241

OPAL $\sqrt{s}=189 \mathrm{GeV}$

DATA $0.210 \pm 0.033 \pm 0.016$

expected $\quad 0.257 \pm 0.004$

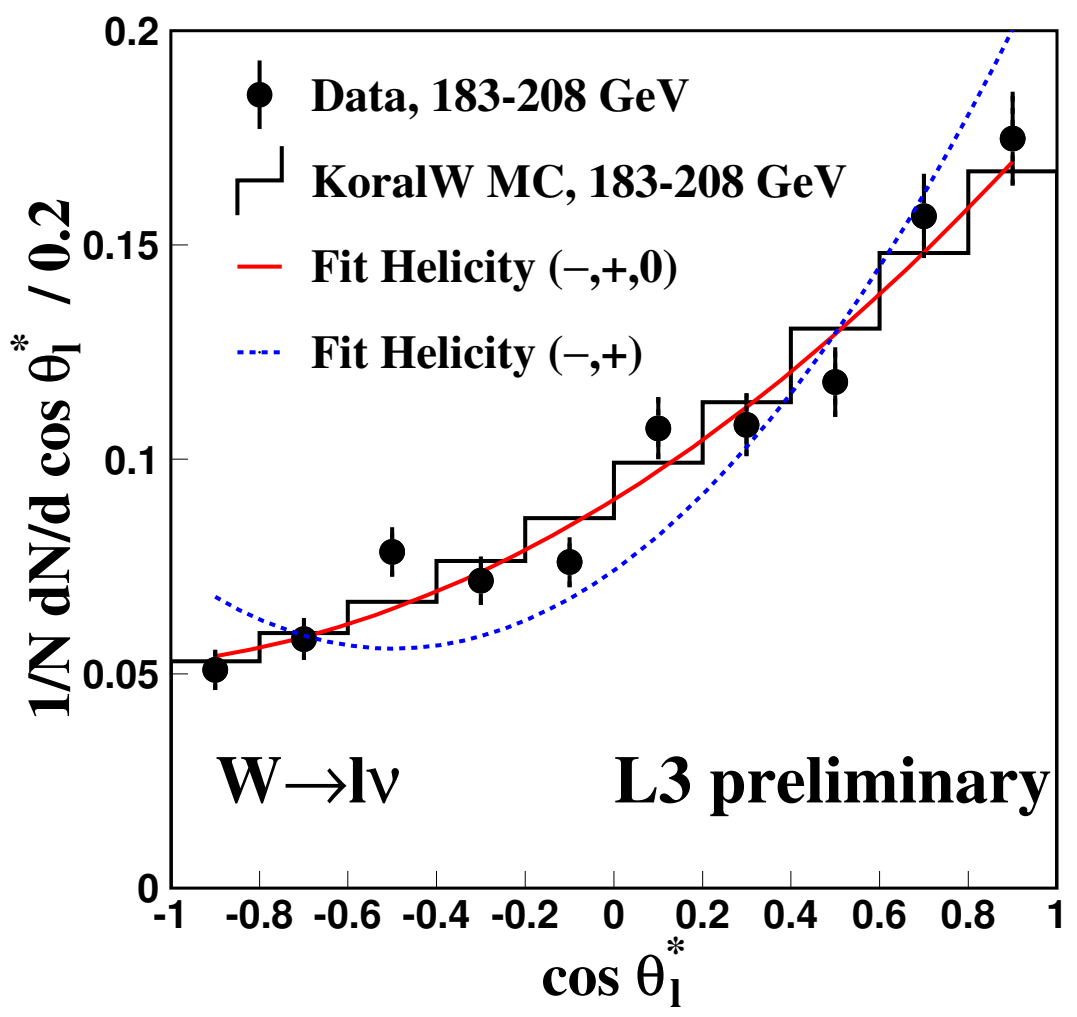

- Fit without longitudinal component fails to describe the data 


\section{Spin Density Matrix}

SDM: normalized product of helicity amplitudes

$$
\rho_{\tau_{-} \tau_{-}^{\prime} \tau_{+} \tau_{+}^{\prime}}\left(\cos \theta_{\mathrm{W}}\right)=\frac{\sum_{\lambda} F_{\tau_{-} \tau_{+}}^{(\lambda)}\left(F_{\tau_{-}^{\prime} \tau_{+}^{\prime}}^{(\lambda)}\right.}{\sum_{\lambda \tau_{+} \tau_{-}}\left|F_{\tau_{-}}^{(\lambda)} \tau_{+}\right|^{2}}
$$

$\tau_{ \pm}$: helicity of $\mathrm{W}^{ \pm} \lambda$ : spin of $\mathrm{e}^{+} \mathrm{e}^{-}$-system

hermitic: 80 independent real coefficents

$\sum \mathbf{W}^{+} \Rightarrow$ single particle matrix element $\mathbf{W}^{-}$

$$
\rho_{\tau_{-} \tau_{-}^{\prime}}^{\mathrm{W}^{-}}\left(\cos \theta_{\mathrm{W}}\right)=\sum_{\tau_{+}} \rho_{\tau_{-} \tau_{-}^{\prime} \tau_{+} \tau_{+}}\left(\cos \theta_{\mathrm{W}}\right)
$$

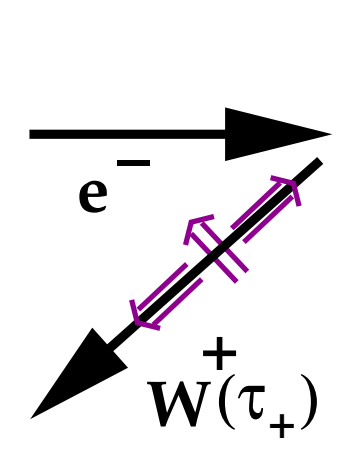

$\Longrightarrow \tau=+1$

$\stackrel{\tau}{\uparrow} \tau=0$

$\Longleftrightarrow \tau=-1$

$\rho_{\tau_{-} \tau_{-}}^{\mathrm{W}_{-}^{-}}$: probability of producing a $\mathrm{W}^{-}$with helicity $\tau_{-}$ 


\section{Spin Density Matrix}

DELPHI preliminary

each helicity amplitude $\longrightarrow$ specific angular symmetry: $\Lambda_{\tau \tau^{\prime}}$ : projection operators

for each bin in $\cos \theta_{\mathrm{W}}$ : $\rho_{\tau \tau^{\prime}}^{W}=$
$\frac{1}{N_{e v .}} \sum_{i}^{e v .} \Lambda_{\tau \tau^{\prime}}\left(\cos \theta^{*}, \phi^{*}{ }_{i}\right)$

- 9 SDM-elements $\rho_{\tau \tau^{\prime}}^{W}$ hermitian matrix $\Rightarrow 6$ independent parameters

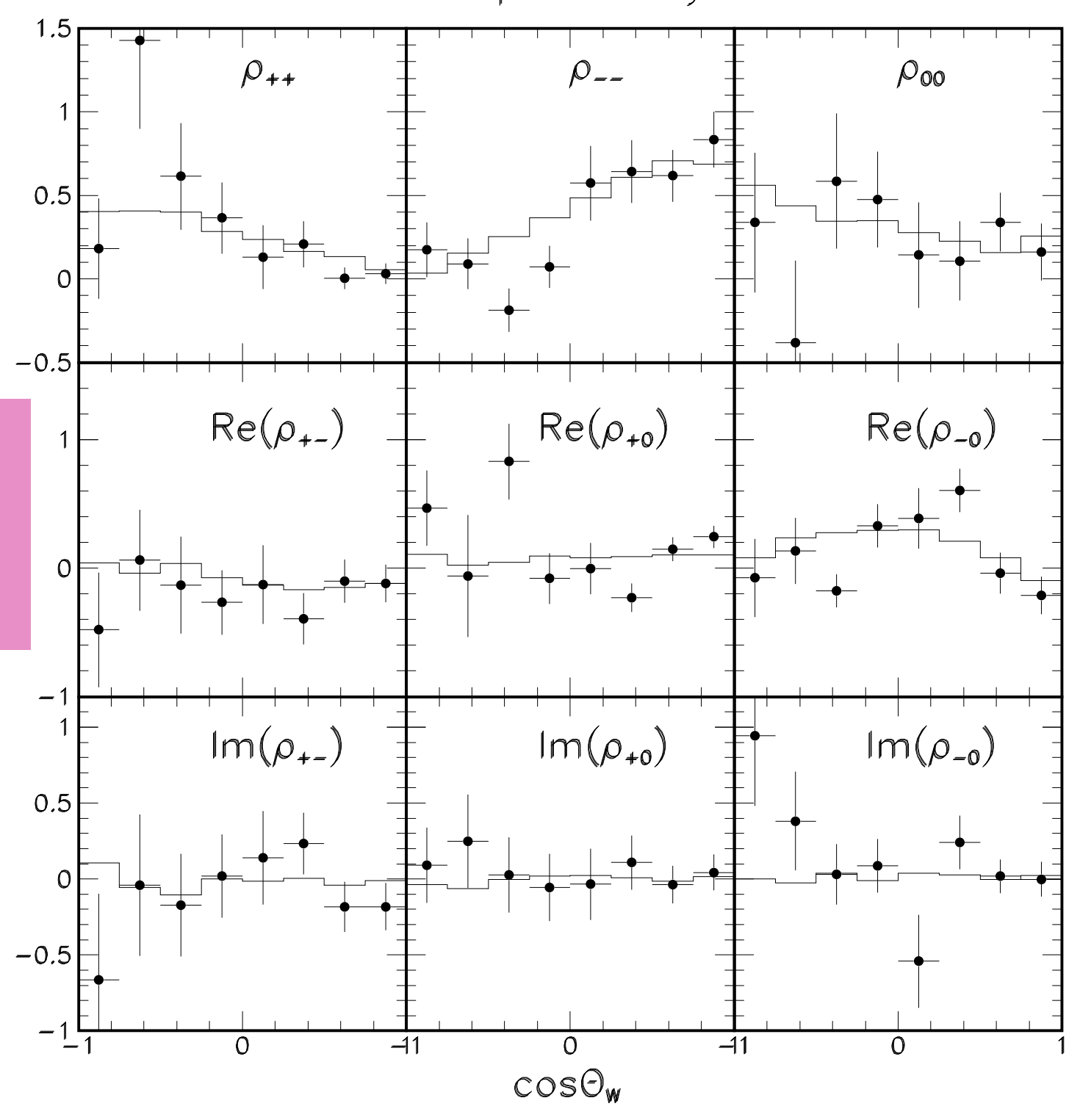




\section{Polarised Differential W Cross Sections}

$\rho_{\tau_{-} \tau_{-}}\left(\cos \theta_{\mathrm{W}}\right) \Rightarrow$

e.g.

longitudinal diff. cross section:

$$
\frac{d \sigma_{L}}{d \cos \theta_{W}}=\rho_{00} \frac{d \sigma}{d \cos \theta_{W}}
$$

OPAL
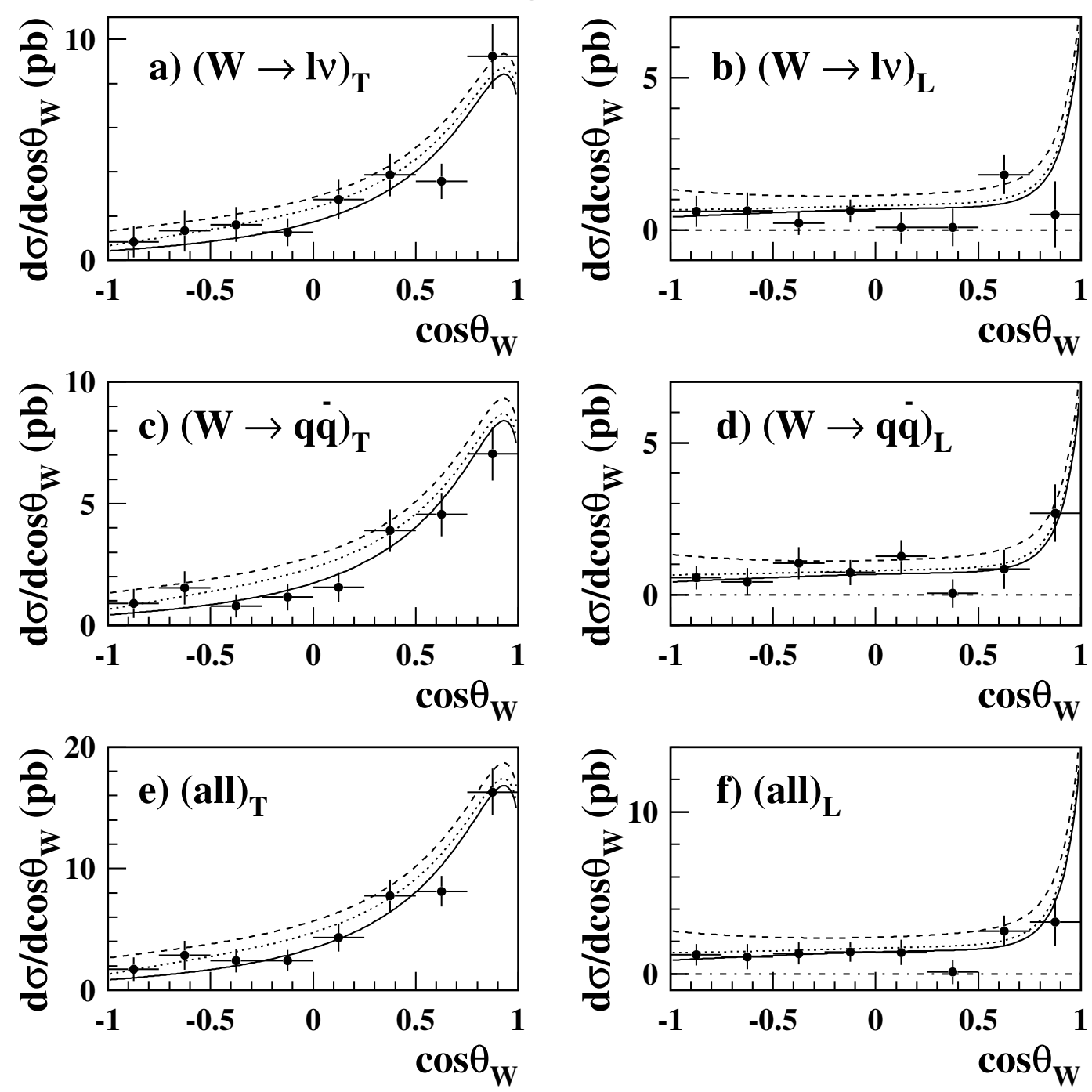


\section{Polarised Differential W Cross Sections}

L3 preliminary

helicity composition in 4

$\cos \theta_{\mathrm{W}}$ regions

- negative $\cos \theta_{\mathrm{W}}$ (backward)
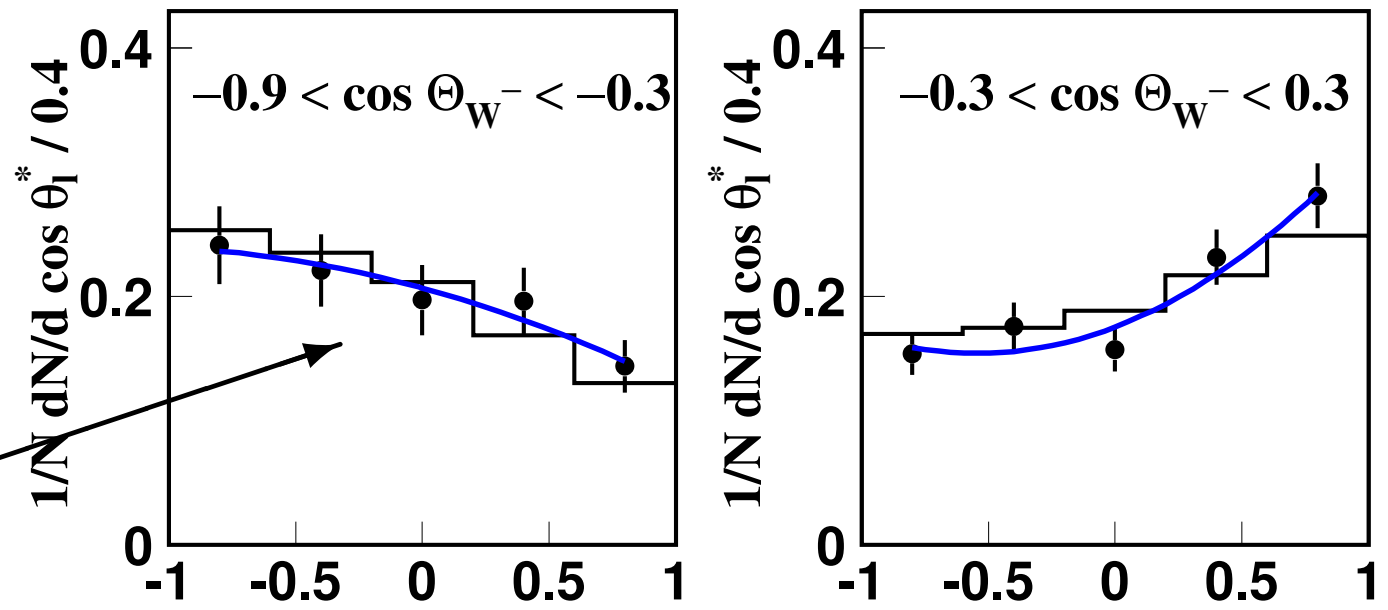

$\longrightarrow\left(\tau_{-}=+1, \tau_{+}=-1\right)$ large

- positive $\cos \theta_{\mathrm{W}}$ (forward)

$\longrightarrow\left(\tau_{-}=-1, \tau_{+}=+1\right)$ large
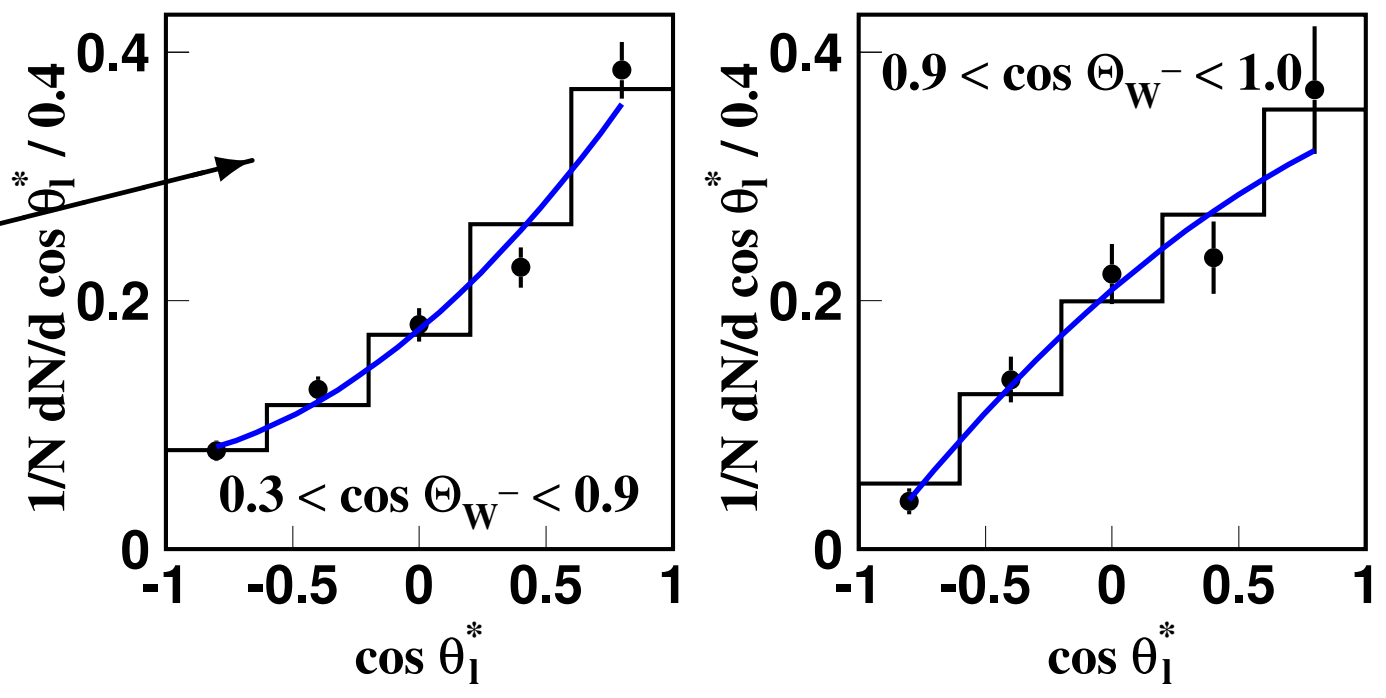


\section{Spin Correlations}

- does the helicity of $\mathrm{W}_{2}$ depend on the helicity of $\mathrm{W}_{1}$ ? fractions of $\left(\tau^{\mathrm{W}^{-}}, \tau^{\mathrm{W}^{+}}\right)=(-,+),(-, 0),(0,0), \ldots$

- enrich (deplete) helicity $\tau= \pm 1$ of $\mathrm{W} \rightarrow \mathrm{q} \overline{\mathrm{q}}$

- measure helicity of $\mathrm{W} \rightarrow \ell \bar{\nu}_{\ell}$
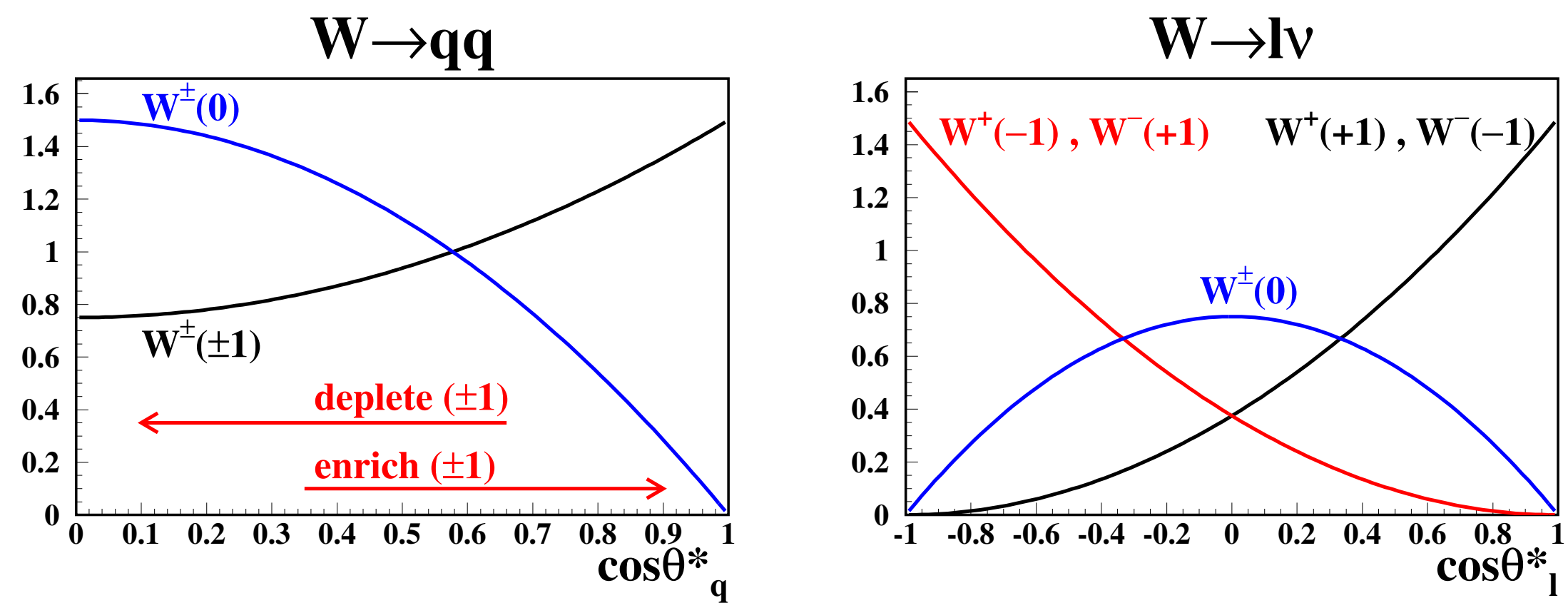


\section{Measurement of Spin Correlations}

- $\cos \theta_{\mathrm{W}}$ region with small (large) (transverse,transverse) W-pair helicity fraction

- "select" $\cos \theta_{\mathrm{q}}^{*}$ region to deplete (enrich) transverse helicity in $\mathrm{W} \rightarrow \mathrm{q} \overline{\mathrm{q}}$

helicity fractions L3 $0.3<\cos \theta_{\mathrm{W}}<0.9$

\begin{tabular}{c|ccc}
\hline & $\tau=-1$ & $\tau=+1$ & $\tau=0$ \\
\pm 1 depleted & $0.46 \pm 0.10 \pm 0.06$ & $0.11 \pm 0.06 \pm 0.04$ & $0.44 \pm 0.12 \pm 0.07$ \\
\pm 1 enriched & $0.83 \pm 0.09 \pm 0.00$ & $0.09 \pm 0.03 \pm 0.03$ & $\mathbf{0 . 0 8} \pm 0.08 \pm 0.03$ \\
difference & $-0.38 \pm 0.13 \pm 0.06$ & $\mathbf{0 . 0 1} \pm 0.07 \pm 0.05$ & $\mathbf{0 . 3 6} \pm 0.14 \pm 0.08$
\end{tabular}
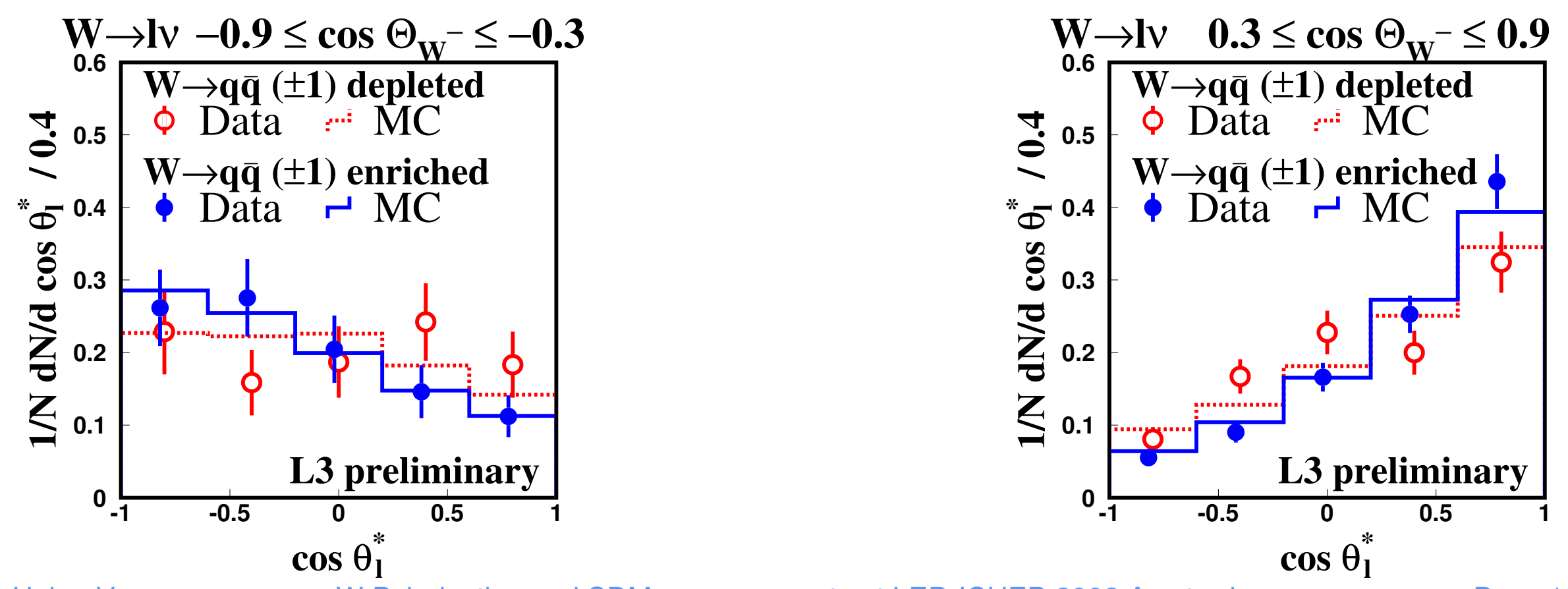


\section{Joint Polarised Cross Sections}

some combinations of the full SDM elements are symmetric in $q \leftrightarrow \bar{q}^{\prime}$

$$
\begin{array}{cc}
\left(\rho_{++++}+\rho_{++--}+\rho_{--++}+\rho_{----}\right) & \propto \sigma_{T T} \\
\left(\rho_{0000}\right) & \propto \sigma_{L L} \\
\left(\rho_{++00}+\rho_{--00}+\rho_{00++}+\rho_{00--}\right) & \propto \sigma_{T L}
\end{array}
$$

\begin{tabular}{c|cc} 
& measured & expected \\
\hline$\frac{\sigma_{\mathrm{TT}}}{\sigma_{\text {total }}}$ & $0.78 \pm 0.09 \pm 0.03$ & $0.57 \pm 0.01$ \\
$\frac{\sigma_{\mathrm{LL}}}{\sigma_{\text {total }}}$ & $0.20 \pm 0.07 \pm 0.02$ & $0.09 \pm 0.01$ \\
$\frac{\sigma_{\mathrm{TL}}}{\sigma_{\text {total }}}$ & $0.02 \pm 0.15 \pm 0.04$ & $0.34 \pm 0.02$
\end{tabular}

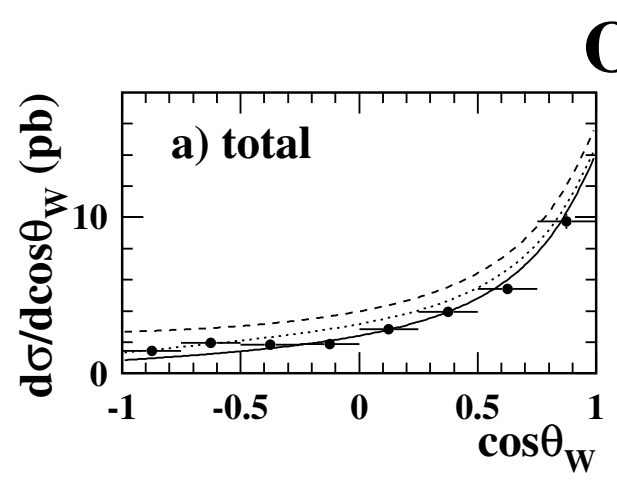

OPAL
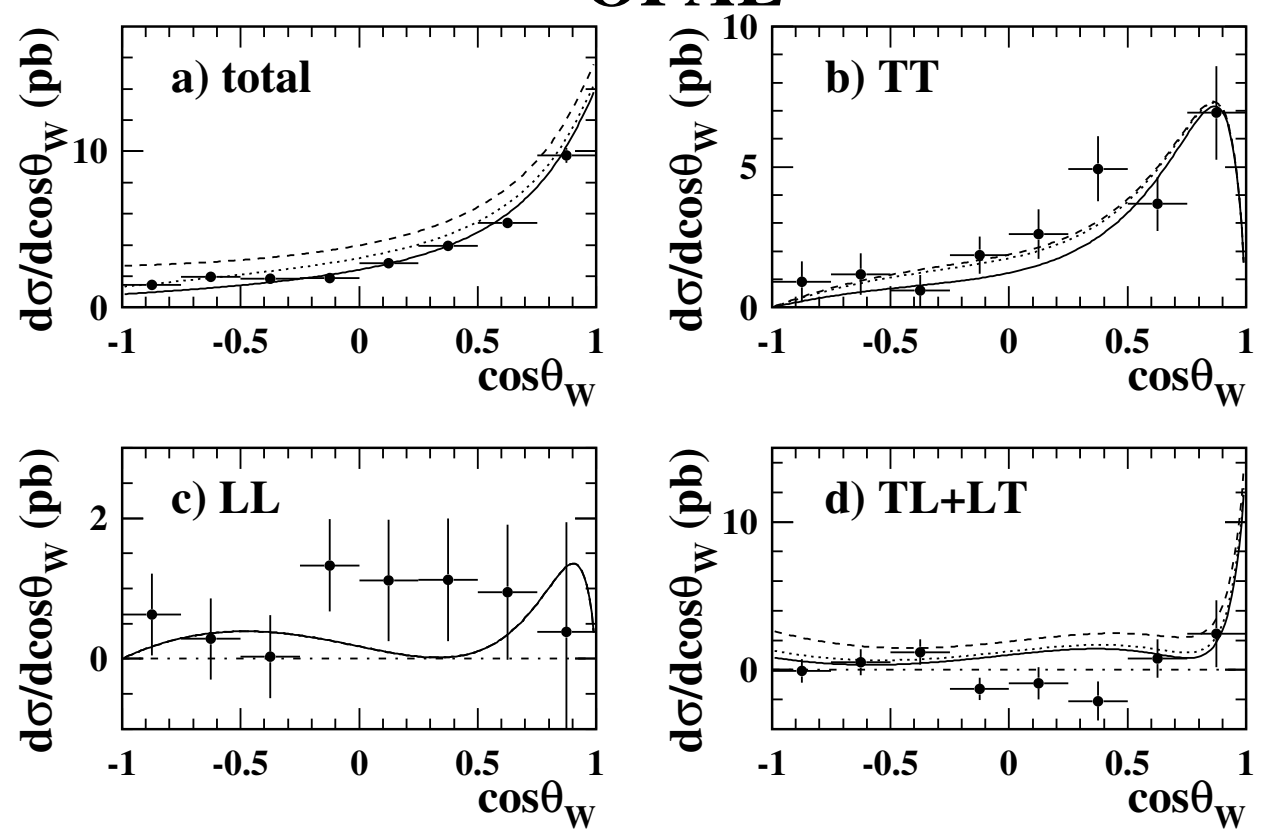


\section{Testing CP Conservation}

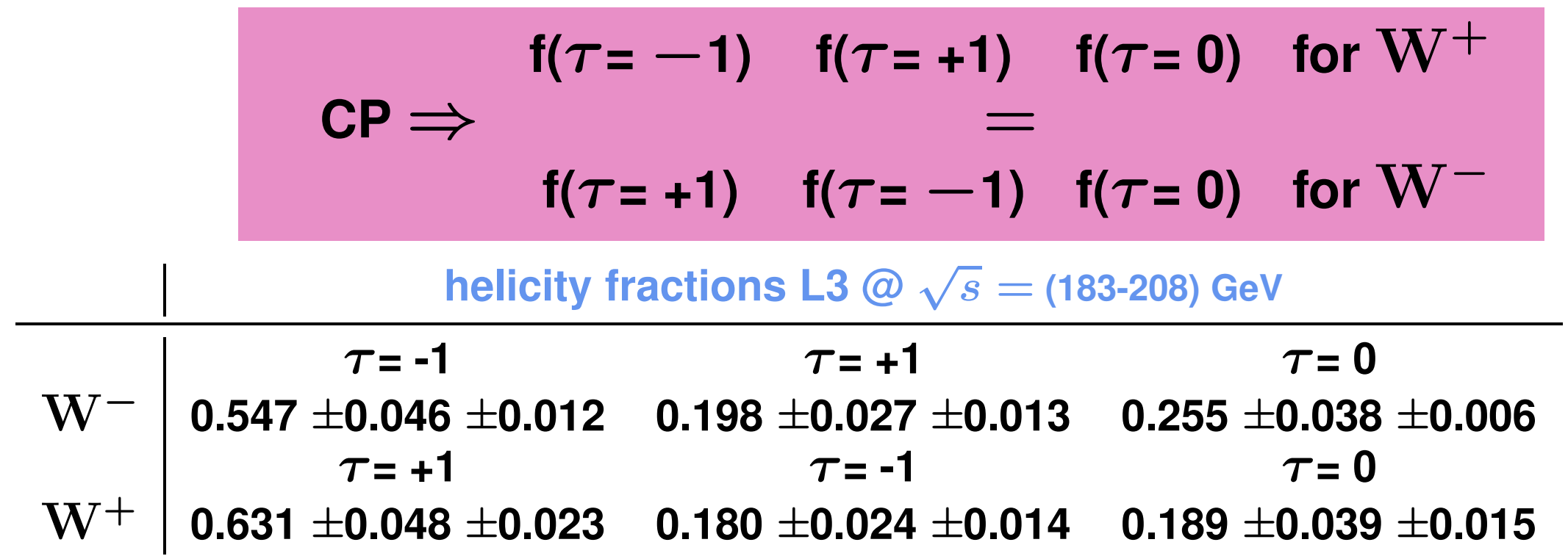

DELPHI, L3, OPAL @ $\sqrt{s}=189 \mathrm{GeV}$

$$
\begin{array}{ll}
\text { CPT: } & \rho_{\tau \tau^{\prime}}^{\mathrm{W}^{+}}=\left(\rho_{-\tau-\tau^{\prime}}^{\mathrm{W}^{-}}\right)^{*} \\
\mathrm{CP}: & \rho_{\tau \tau^{\prime}}^{\mathrm{W}^{+}}=\rho_{-\tau-\tau^{\prime}}^{\mathrm{W}^{-}}
\end{array}
$$

$\mathrm{CPT}+\mathrm{CP} \Rightarrow \rho^{\mathrm{W}}$ are real 


\section{Test of CP-Violation}

L3 Preliminary

$\mathrm{CP}$ violation at tree level:

$$
\operatorname{Im}\left(\rho_{\tau \tau^{\prime}}^{\mathrm{W}^{+}}\right)-\operatorname{Im}\left(\rho_{-\tau-\tau^{\prime}}^{\mathrm{W}^{-}}\right) \neq 0
$$

CP violation at loop level or

CPT violation at tree level:

$$
\operatorname{Im}\left(\rho_{\tau \tau^{\prime}}^{\mathrm{W}^{+}}\right)+\operatorname{Im}\left(\rho_{-\tau-\tau^{\prime}}^{\mathrm{W}^{-}}\right) \neq 0
$$
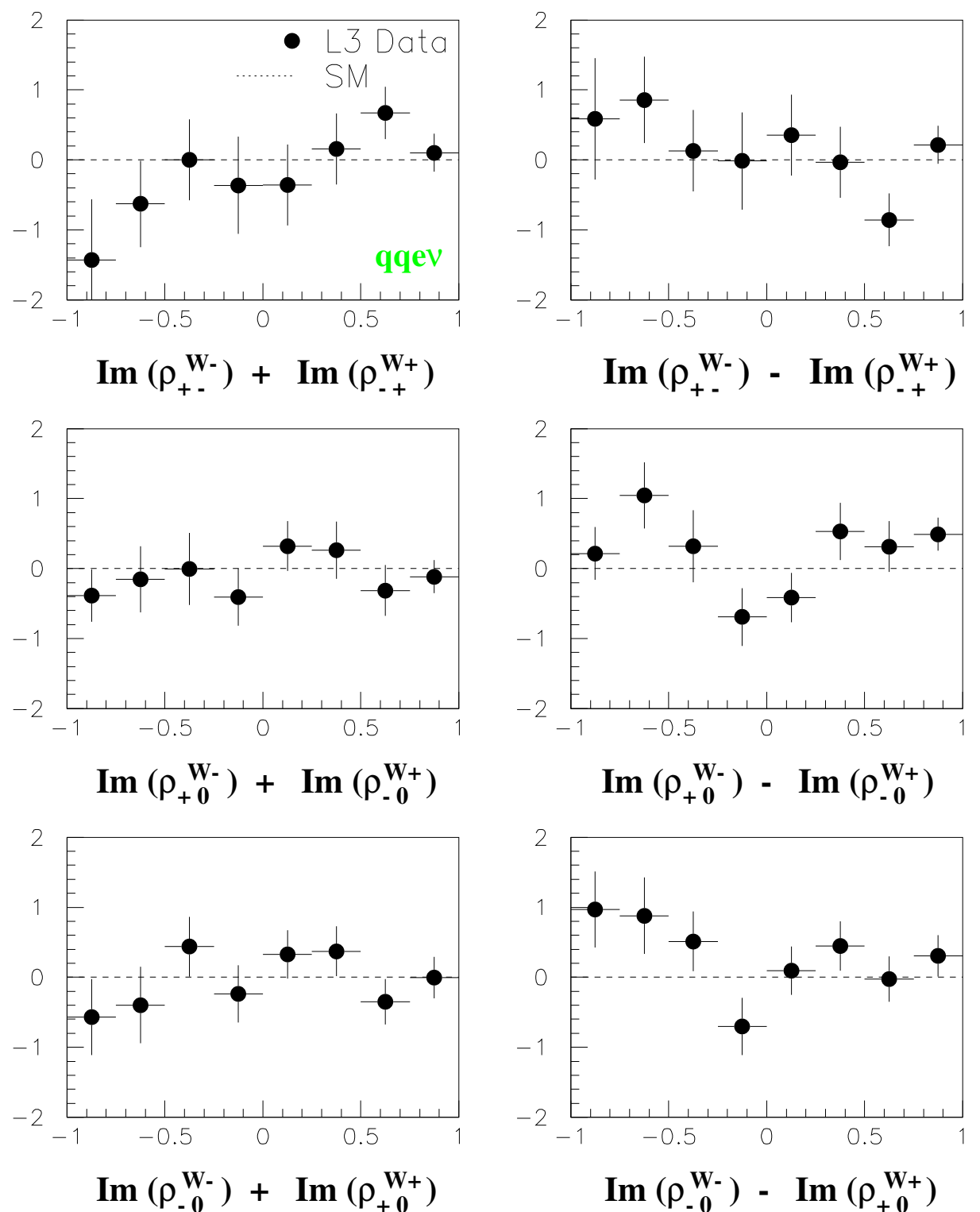

$$
\operatorname{Im}\left(\rho_{-0}^{\mathrm{W}-}\right)+\operatorname{Im}\left(\rho_{+\mathbf{0}}^{\mathrm{W}+}\right)
$$$$
\cos \theta_{W}
$$

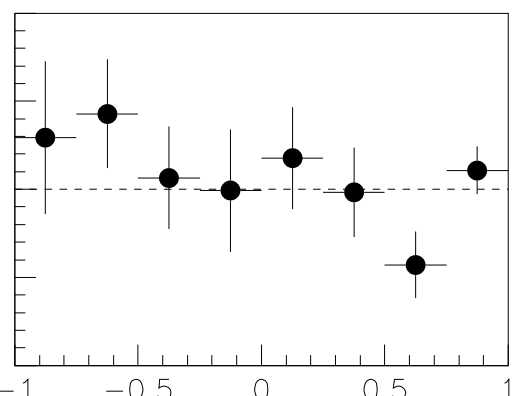

$$
\operatorname{Im}\left(\rho_{+-}^{\mathbf{W}}\right)-\operatorname{Im}\left(\rho_{-+}^{\mathbf{W +}}\right)
$$

$$
\operatorname{Im}\left(\rho_{+\mathbf{0}}^{\mathbf{W}-}\right)-\operatorname{Im}\left(\rho_{-\mathbf{0}}^{\mathrm{W}+}\right)
$$

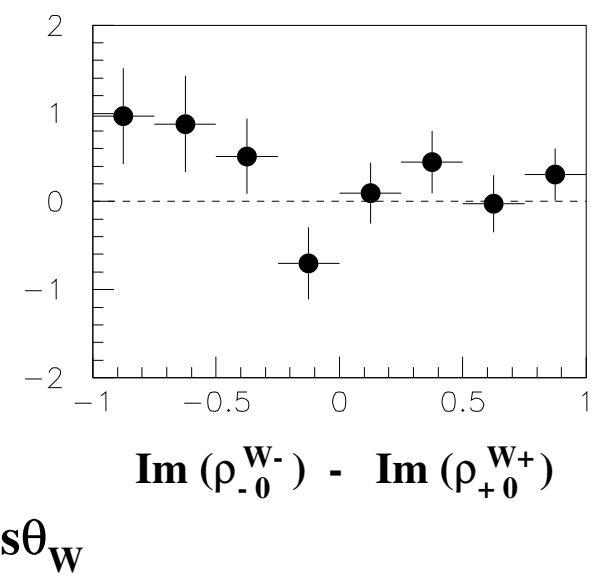




\section{CP-Violating Triple Gauge Couplings}

- fitting SDM elements to

MC expectations (OPAL)

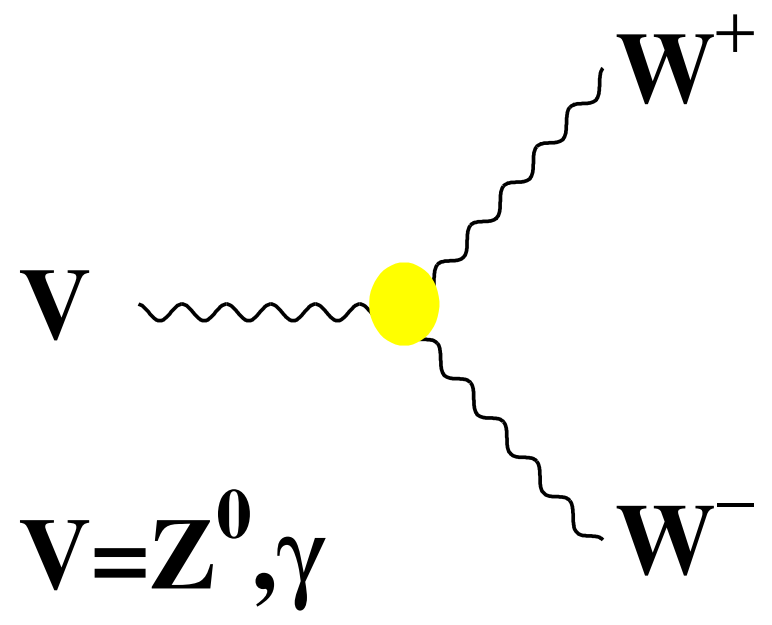

\begin{tabular}{c|ccc} 
& $\tilde{\kappa}_{Z}$ & $g_{4}^{Z}$ & $\tilde{\lambda}_{Z}$ \\
\hline SDM elements & $-0.19_{-0.07}^{+0.08}$ & $0.00_{-0.20}^{+0.21}$ & $-0.12_{-0.16}^{+0.17}$ \\
$\cos \theta_{\mathrm{W}}$ & $-0.19_{-0.08}^{+0.46}$ & $0.7_{-1.8}^{+0.4}$ & $-0.29_{-0.11}^{+0.69}$ \\
combined (+sys) & $-0.20_{-0.07}^{+0.10}$ & $-0.02_{-0.33}^{+0.32}$ & $-0.18_{-0.16}^{+0.24}$
\end{tabular}

there are other measurements of CP-violating TGCs by ALEPH, DELPHI that use different methods 


\section{Summary}

- ee $\rightarrow$ WW spin structure from DELPHI, L3, OPAL

- polarised $\mathrm{W}$ cross section measured longitudinal W-Bosons confirmed

- Spin Density Matrix analysis (more data analysed soon)

- correlated helicity states studied

- CP, CPT tests (and CP - violating TGCs) 